University of Wollongong

Research Online

Faculty of Health and Behavioural Sciences -

Papers (Archive)

Faculty of Science, Medicine and Health

2010

\title{
Molecular cloning and characterization of the global regulator LaeA in
}

Penicillium citrinum

Wei Xing

Southwest University

Chao Deng

University of Wollongong, chao@uow.edu.au

Chang-Hua $\mathrm{Hu}$

Southwest University

Follow this and additional works at: https://ro.uow.edu.au/hbspapers

Part of the Arts and Humanities Commons, Life Sciences Commons, Medicine and Health Sciences

Commons, and the Social and Behavioral Sciences Commons

\section{Recommended Citation}

Xing, Wei; Deng, Chao; and Hu, Chang-Hua: Molecular cloning and characterization of the global regulator LaeA in Penicillium citrinum 2010.

https://ro.uow.edu.au/hbspapers/3460

Research Online is the open access institutional repository for the University of Wollongong. For further information contact the UOW Library: research-pubs@uow.edu.au 


\title{
Molecular cloning and characterization of the global regulator LaeA in Penicillium citrinum
}

\author{
Abstract \\ We have cloned and analysed a laeA gene (Pci-laeA) that may control mevastatin biosynthesis in \\ Penicillium citrinum. The full-length Pci-laeA sequence is 1,340 bp with an ORF of 1,284 bp encoding 427 \\ amino acids. It shows $95 \%$ identity with LaeA from P. chrysogenum. The predicted molecular mass of Pci- \\ LaeA is $48.72 \mathrm{kDa}$ with an estimated theoretical isoelectric point of 6.96. Pci-LaeA has a conserved S- \\ adenosylmethionine binding site and a potential MlcR (a pathway specific regulator in mevastatin \\ biosynthesis) binding site.

\section{Disciplines} \\ Arts and Humanities | Life Sciences | Medicine and Health Sciences | Social and Behavioral Sciences

\section{Publication Details} \\ Xing, W., Deng, C. \& Hu, C. (2010). Molecular cloning and characterization of the global regulator LaeA in \\ Penicillium citrinum. Biotechnology Letters, 32 (11), 1733-1737.
}




\section{Molecular cloning and characterization of the global regulator LaeA in Penicillium citrinum}

Wei Xing ${ }^{1}$, Chao Deng ${ }^{2}$, Chang-Hua $\mathrm{Hu}^{1,2, *}$

1. Institute of Modern Biopharmaceuticals, School of Pharmaceutical Sciences, Southwest University, Beibei, Chongqing 400716, People Republic of China

2. School of Health Sciences, University of Wollongong, Wollongong, 2522, NSW, Australia

*Corresponding author.

Prof Chang-Hua Hu, Institute of Modern Biopharmaceuticals, School of Pharmaceutical Sciences, Southwest University, Beibei, Chongqing 400716, People Republic of China

Fax: +862368251225

E-mail address: chhhu@swu.edu.cn $(\mathrm{C}-\mathrm{H}$. Hu)

The section to be selected: Microbial and enzyme biotechnology 


\begin{abstract}
LaeA, a novel global regulator of secondary metabolism, has been discovered in filamentous ascomycetes. In this study, we have cloned and analysed a laeA gene (Pci-laeA) which may control mevastatin biosynthesis in Penicillium citrinum. The full-length Pci-laeA sequence is $1340 \mathrm{bp}$ in size, and contains an open reading frame of 1284 bp encoding 427 amino acid, which shows $95 \%$ identity with that of $P$. chrysogenum LaeA found in 2008. The predicted molecular mass of Pci-LaeA is 48.72 $\mathrm{kDa}$ with an estimated theoretical isoelectric point of 6.96. Pci-LaeA shows a conserved S-adenosylmethionine binding site and a potential MlcR (a pathway specific regulator in mevastatin biosynthesis) binding site.
\end{abstract}

Keywords: Cloning; Global regulator LaeA; Mevastatin; Penicillium citrinum 


\section{Introduction}

Mevastatin (compactin), the first potent and specific competitive inhibitor of 3-hydroxy-3-methylglutaryl-coenzyme A (HMG-CoA) reductase, is a polyketide compound produced by Penicillium citrinum, and has been used as a substrate for microbial conversion to pravastatin, a widely used pharmaceutical product for the therapy of hypercholesterolemia (Endo et al. 1976, Park et al. 2003). The gene cluster responsible for compactin biosynthesis in P. citrinum has been identified (Abe et al. 2002). Among them, $m l c R$ is a pathway-specific zinc cluster transcriptional activator of the compactin biosynthetic genes( Baba et al. 2009 ).

In addition to pathway-specific regulators, fungal secondary metabolite biosynthesis is also regulated by global regulators (Keller et al. 2005). LaeA, a global regulator for morphological development and secondary metabolism in filamentous fungi has been identified (Bok and Keller 2004). In Aspergillus nidulans, where LaeA was first discovered, deletion of laeA resulted in a loss of production of numerous secondary metabolites, such as sterigmatocystin and penicillin (Bok and Keller 2004). Moreover, the production of several toxins was regulated by LaeA in Aspergillus fumigatus (Bok et al. 2005). In Penicillium chrysogenum, LaeA positively regulated penicillin biosynthesis, the formation of pigmentation and sporulation (Kosalkova et al. 2008). Therefore, it is important to determine the role of laeA in the secondary metabolism of the mevastatin producer P. citrinum. In this work, we identified and characterized a $P$. citrinum laeA (Pci-laeA) gene encoding a protein very similar to that of Penicillium chrysogenum, A. fumigatus and A. nidulans. The discovery of Pci-laeA will facilitate elucidation of the regulatory mechanisms in mevastatin biosynthesis and contribute to strain improvement and the discovery of novel natural products. 


\section{Materials and Methods}

\section{Strains, media and culture conditions}

Penicillium citrinum IMB002, a high-level mevastatin producer, was collected in our laboratory.

P. citrinum liquid cultures were initiated by inoculating fresh spores inoculated into complex medium

(10 g tryptone/1, $50 \mathrm{~g}$ glucose/1, $30 \mathrm{~g}$ soybean powder/l, $10 \mathrm{~g}$ glycerol/1, $5 \mathrm{~g} \mathrm{NaNO}_{3} / 1,1 \mathrm{~g} \mathrm{MgSO} / \mathrm{l}, \mathrm{pH}$

6.5). After incubation at $25{ }^{\circ} \mathrm{C}$ for $72 \mathrm{~h}$ in an orbital shaker $(210 \mathrm{rpm})$, aliquots were inoculated in complex mevastatin production medium CMPM (10 g tryptone/1, $50 \mathrm{~g}$ glucose/1, $35 \mathrm{~g}$ soybean powder/l, $50 \mathrm{~g}$ glycerol/1, $5 \mathrm{~g} \mathrm{NaNO}_{3} / 1,1 \mathrm{~g} \mathrm{MgSO}_{4} / 1,2.6 \mathrm{~g}$ bean oil/1, $\mathrm{pH} 6.5$ ) at $25^{\circ} \mathrm{C}$.

\section{Extract of genomic DNA and isolation of total RNA}

Genomic DNA from Penicillium citrinum was isolated from mycelium grown for 96h in CMPM medium using the CTAB method. Total RNA was also isolated from mycelium grown for $96 \mathrm{~h}$ in CMPM medium using the 'RNA-prep Pure Plant Kit' (Tiangen, China), following the manufacturer's instructions.

\section{RT-PCR and cloning of the internal fragment of $P$. citrinum laeA cDNA}

Based on the known amino acid sequences of several LaeA proteins deposited in the NCBI database, the conserved region of the laeA gene was identified by MegAlign program of DNAstar software. Two

degenerate primers D1: 5'-GARCARGAYCGYCTNGAYWT-3' and D2:5' GCYTGRTAKATRTGMAG- 3' were synthesized to amplify the internal fragment of P. citrinum laeA cDNA by RT-PCR using the 'Takara RNA PCR Kit' (Takara, Japan), following the manufacturer's instruction.

\section{Cloning of ' 3' end sequences of the laeA using RACE technique}

To obtain the $3^{\prime}$-end sequences of laeA cDNA, the rapid amplification of cDNA ends (RACE) 
technique was used. The oligonucleotide $\mathrm{d}(\mathrm{T}) 18$-adaptor reverse primer: 5'-TACCGTCGTTCCACTAGTGATTTCACTATAGGTTTTTTTTTTTTTTTTTT-3' was used for the synthesis of the first-strand cDNA. The adaptor reverse primer: 5'-TACCGTCGTTCC ACTAGTGATTT-3' and the gene-specific primer F1: 5'-CCCGTGTTGAGGACAAGGATGGT-3' were used for amplification of cDNA. Subsequently, the PCR product was tested by two consecutive nested PCR reactions with the adaptor reverse primer and gene-specific primers F2: 5 '-CTGATTCCCA CGAGCAGAAGGTT-3', and with the adaptor reverse primer and gene-specific primers F3: 5'CAGTCGGGTTCTTTCTTGGTCC-3', respectively. .

\section{Rapid isolation of 5'-flanking region of laeA by TAIL-PCR}

Cloning of the $5^{\prime}$ flanking region of laeA was carried out by TAIL-PCR (thermal asymmetric interlaced PCR) technique with some modifications (Liu and Whittier 1995): (1) 10 mers arbitrary primers AD1: 5'- GNANCANAGA-3', AD2: 5'- GNANCANAGG-3', AD3: 5'- CNGANANGAA-3' and AD4: 5' GNACNTNGGA-3' were used; (2) 10 low-stringency cycles were deleted; and (3) the tertiary PCR procedure was the same as the secondary TAIL-cycling (15 super cycles) instead of normal cycles. Three gene-specific reverse primers R1: 5'-GTCAACACCGACCACGAAGGAAT-3', R2: 5'-GATGCTGAAAGACTCGTCGGTAG-3' and R3: 5'-CAAGAAAGAACCCGACTGAAAGG-3' were obtained against the cloned internal fragment of laeA cDNA.

\section{Cloning of the whole $P$. citrinum laeA gene}

Assembly of the internal and flanking fragments was carried out to form the full-length sequence of the laeA gene using the SeqMan program of DNAstar software. The primers LAF1: 5'-AATATCAACGGATCCATGTCTTACAGAGAG-3' and LAR1: 5'-TCACCAGGG AGCTCTTATTCCTCGAGAGG-3' were used to amplify the coding region of laeA cDNA. 


\section{Bioinformatics analysis of the $P$. citrinum LaeA sequence}

The molecular mass and $\mathrm{pI}$ (isoelectric point) of P. citrinum LaeA (Pci-LaeA) protein was determined using ProtParam tools (http://www.expasy.org/tools/protparam.html). The sequence homology was analyzed by BlastP of NCBI (http://www. ncbi. nlm. nih. gov). The alignment of amino acid sequences was carried out using the ClustalW2 (http://www.ebi.ac.uk/clustalw/). The prediction concerning subcellular localization of the protein was performed using WoLF PSORT software

(http://wolfpsort.org/).

\section{Accession number}

The full length genomic DNA and cDNA sequences of the Pci-laeA gene were submitted to the GenBank database under the accession numbers GU647101 and GU906782 respectively.

\section{Results and Discussion}

\section{Cloning and analysis of full length DNA and cDNA of Pci-laeA}

Using degenerate primers D1 and D2, a 782-bp cDNA fragment was obtained from P. citrinum by RT-PCR (Fig. 1A). By 3'RACE and TAIL-PCR technique, we obtained the 3' end fragment (1136 bp) and a 5 '-flanking fragment (981 bp) of Pci-laeA (Fig. 1B and Fig. 1C). The whole laeA gene (1340 bp) and the encoding gene laeA (1284 bp) gene were amplified from the genomic DNA and cDNA of $P$. citrinum by PCR using primers LAF1 and LAR1, respectively (Fig. 1D). A 56-bp intron spanning nucleotides 390-445 of the Pci-laeA gDNA were identified. Sequences of the 5' and 3' intron splice sites were 'GTATGT' and 'GAACAG'respectively. These concur with the consensus fungal intron splice sequences.

An examination of the laeA nucleotide sequence revealed one putative MlcR binding site 
5'-ACGG-TTGTCGTGA-TCGG-3' that was very similar to the consensus sequence for the MlcR-binding sequence 5'-(A/T)CGG-NGTN3-6- TCGG-3' (Baba et al. 2009). It has been recongnised that the mlcR binding sites motif is an asymmetric direct repeat that exists in the $m l c A-m l c C, m l c B-m l c D, m l c F-m l c G$ and $m l c H$-orf11 intergenic regions, and is essential for the transcriptional activation of $m l c A$ and $m l c C$ in P. citrinum (Baba et al. 2009). Other similar studies have revealed that another GAL-4 type pathway transcriptional activator AflR has binding sites in the sterigmatocystin (ST) cluster gene and laeA gene simultaneously in A. nidulans (Fernandes et al. 1998, Bok and Keller 2004). Overexpression of the A. nidulans laeA upregulated alfR expression, while overexpression of alfR downregulated laeA expression (Bok and Keller 2004). This implies that there may be a potential mlcR binding site in the $P$. citrinum laeA gene promoter and a unique interaction between laeA and mevastatin gene regulation.

\section{Characterization of $P$. citrinum LaeA protein and homology analysis}

The Pci-laeA encoded a protein of 427 amino acids with an estimated molecular mass of $48.72 \mathrm{kDa}$ and a theoretical pI of 6.96. The results of sequence homology analysis indicated that Pci-LaeA showed $95 \%, 61 \%$ and $60 \%$ identity with LaeA protein of $P$. chrysogenum, A. fumigatus and A. nidulans, respectively. Aligment of the amino acid sequences of the Pci-LaeA protein revealed a conserved S-adenosylmethionine (SAM) binding motif (Fig. 2B), component of the 193rd to 200th amino acid

residues (- LDLGCGTG -), the 219th and 239th amino acid residues of P. citrinum LaeA protein, normally found in most of the protein methyltransferase (Hamahata et al. 1996). Although Pci-LaeA contains the same SAM motif in histone methyltransferases and arginine methyltransferases as LaeA protein found in other species, it lacks two other conserved domains (SET domain and a double E loop). Therefore, Pci-LaeA is possibly a unique protein methyltransferase. This remains to be elucidated 
through further study.

The Pci-LaeA protein was analyzed using WoLF PSORT software. The analysis showed that this protein was localized in the nucleus, however it did not contain obvious nuclear localization signal sequence. This phenomenon has also been observed in A. nidulans LaeA. In A. nidulans, the LaeA did not show the presence of a nuclear localization motif, however the existence of GFP tagging in either the $5^{\prime}$ or $3^{\prime}$ ends suggested that LaeA should be primarily localized in the nucleus (Bok and Keller 2004). Due to its nuclear localization and conserved methyltransferase SAM-binding motif, the Pci-LaeA protein should be correlated with a change in chromatin conformation (Bok and Keller 2004,).

The discovery of Pci-LaeA provides an important clue for understanding the secondary metabolism of mevastatin. As demonstrated by transcriptome microarry comparative analysis between wild-type, a LaeA deletion mutant and complemented control strains of A. fumigatus, LaeA modulates the expression of approximate $10 \%$ of the genes in A. fumigatus. Furthermore, 13 secondary metabolite clusters including 102 genes are under the control of LaeA (Perrin et al. 2007). Overexpression of laeA has been reported to enhance expression of some secondary metabolic gene clusters and consequently increased products formation. For example, there was $400 \%$ to $700 \%$ production increase in lovastatin when the A. nidulans laeA gene was heterogeneously expressed in the A. terreus (Bok and Keller 2004). In addition, over-expression of the PclaeA gene could lead to a $25 \%$ increase in penicillin production in P. chrysogenum (Kosalkova et al. 2008). It is even more exciting that modifications of LaeA have been used as a tool to trigger cryptic new natural products, such as terrequione A (an anti-tumor agent that was not previously known to be produced by A. nidulans) (Bouhired et al. 2007). Although the function of LaeA has not been well understood, laeA-based genomic mining has already shown 
strong potential as a strategy for activating silent cryptic biosynthetic gene clusters (Bok et al. 2006; Zerikly et al. 2009). The identification of $P$. citrinum LaeA will be useful for the improvement of industrial strain and the discovery of novel natural products.

\section{Acknowledgments}

This work was supported by the grants of Chongqing Key Program of Science and Technology Development (CSTC2009AB1029). We would like to thank Dr Mandy Reid and Dr Guojian Liao for critical reading of this manuscript.

\section{References}

Abe Y, Suzuki T, Ono C, Iwamoto K, Hosobuchi M (2002) Molecular cloning and characterization of an ML-236B (compactin) biosynthetic gene cluster in Penicillium citrinum. Mol Genet Genomics 267: $636-646$

Baba S, Kinoshita H, Hosobuchi M, Nihira T (2009) MlcR, a zinc cluster activator protein, is able to bind to a single (A/T)CGG site of cognate asymmetric motifs in the ML-236B (compactin) biosynthetic gene cluster. Mol Gene. Genomics 281: 627-634

Bok JW and Keller NP (2004) LaeA, a regulator of secondary metabolism in Aspergillus spp. Eukaryot Cell 3: 527-535

Bok JW, Balajee SA, Marr KA, Andes D, Nielsen KF, Frisvad JC, Keller NP(2005) LaeA, a regulator of morphogenetic fungal virulence factors. Eukaryotic Cell 4: 1574-1582

Bok JW, Hoffmeister D, Maggio-Hall LA, Murillo R, Glasner JD , Keller NP (2006) Genomic mining 
for Aspergillus natural products. Chem Biol 13: 31-37

Bouhired S, Weber M, Kempf-Sontag A, Keller NP, Hoffmeister D (2007) Accurate prediction of the Aspergillus nidulans terrequinone gene cluster boundaries using the transcriptional regulator LaeA. Fungal Genet Biol $44: 1134-1145$

Endo A, Kuroda M and Tanzawa K (1976) Competitive inhibition of 3-hydroxy- 3- methylglutaryl coenzyme A reductase by ML-236A and ML-236B fungal metabolites, having hypocholesterolemic activity. FEBS Lett 72: 323-326

Fernandes M, Keller NP, Adams TH(1998) Sequence-specific binding by Aspergillus nidulans AflR, a C-6 zinc cluster protein regulating mycotoxin biosynthesis. Mol Microbiol 28:1355-1365

Hamahata A, Takata Y, Gomi T, Fujioka M (1996) Probing the S-adenosylmethionine- binding site of rat guanidinoacetate methyltransferase. J Biochem 317 : 141-145

Kale SP, Milde L, Trapp MK, Frisvad JC, Keller NP, J.W. Bok (2008) Requirement of LaeA for secondary metabolism and sclerotial production in Aspergillus flavus. Fungal Genet Biol 45: $1422-1429$

Keller NP, Turner G, Bennett JW, (2005) Fungal secondary metabolism - from biochemistry to genomics. Nat Rev. Microbiol, 3: 937-947

Kosalkova K, Garcia-Estrada C, Ullan RV, Godio RP, Feltrer R (2008) The global regulator LaeA controls penicillin biosynthesis, pigmentation and sporulation, but not roquefortine $\mathrm{C}$ synthesis in Penicillium chrysogenum. Biochimie 91: 214-225

Liu Y-G and Whittier RF (1995) Thermal asymmetric interlaced PCR: automatable amplification and sequencing of insert end fragments from P1 and YAC clones for chromosome walking. Genomics 25: $674-681$ 
Park JW, Lee JK, Kwon TJ , Yi DH, Kim YJ (2003) Bioconversion of compactin into pravastatin by Streptomyces sp. Biotechnol Lett 25: 1827-1831

Perrin RM, Fedorova ND, Bok JW, Cramer RA, Wortman JR (2007) Transcriptional regulation of chemical diversity in Aspergillus fumigatus by LaeA. Plos Pathog 3: 508-517

Zerikly M, Challis GL(2009) Strategies for the discovery of new natural products by genome mining.

Chembiochem $10: 625-633$. 

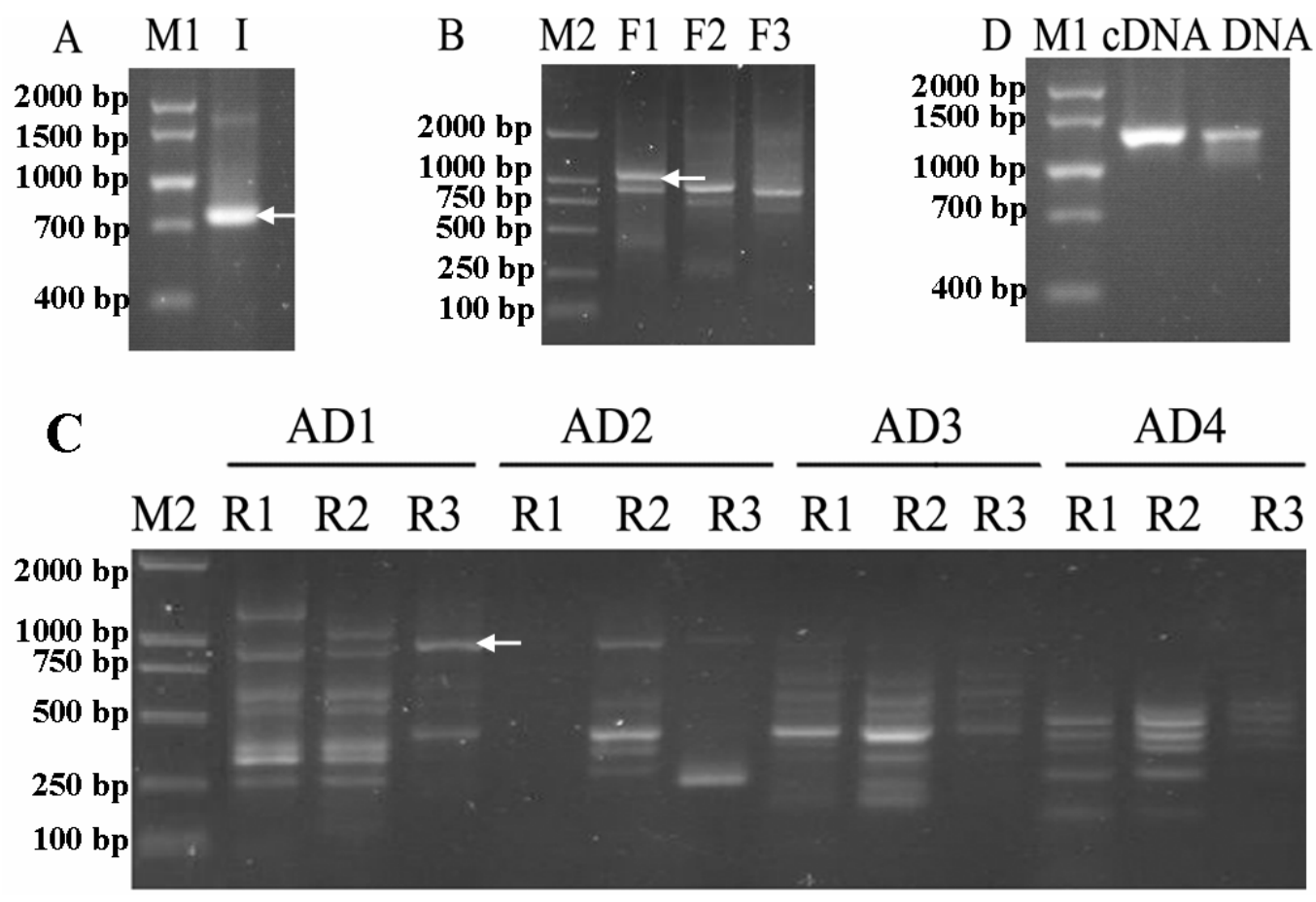

Figure 1 Cloning of the P. citrinum laeA gene. (A) Amplification of the internal fragment of cDNA using degenerate primers D1 and D2. Lane I, The expected PCR fragment indicated by the arrow is 782 bp in size . M1, DNA Marker (2000 bp, 1500 bp, 1000 bp, 700 bp, 400 bp, 200 bp). (B) Amplification of laeA cDNA 3'end by RACE with nested primers F1-3. The expected PCR fragment indicated by the arrow is 1136 bp in size. M2, DNA Marker (2000 bp, 1000 bp, 750 bp, 500 bp, 250 bp, 100 bp). (C) Isolation of 5'-flanking region of laeA by TAIL-PCR using degenerate arbitrary primers AD1-4 and nested gene-specific primers R1-3. The expected PCR fragment indicated by the arrow is $981 \mathrm{bp}$ in size. No expected PCR product was obtained with the primers AD2/R1-3, AD3/R1-3 and AD4/R1-3. M2, DNA marker (2000 bp, 1000 bp,750bp, 500 bp, 250 bp, 100 bp). $\quad$ (D) PCR amplification of the whole Pci-laeA genomic DNA and cDNA using primers LAF1 and LAR1. The Pci-laeA genomic DNA is of $1340 \mathrm{bp}$, and cDNA is of $1284 \mathrm{bp}$. M1, DNA Marker (2000 bp, $1500 \mathrm{bp}, 1000 \mathrm{bp}, 700 \mathrm{bp}, 400 \mathrm{bp}$, $200 \mathrm{bp})$. 
A

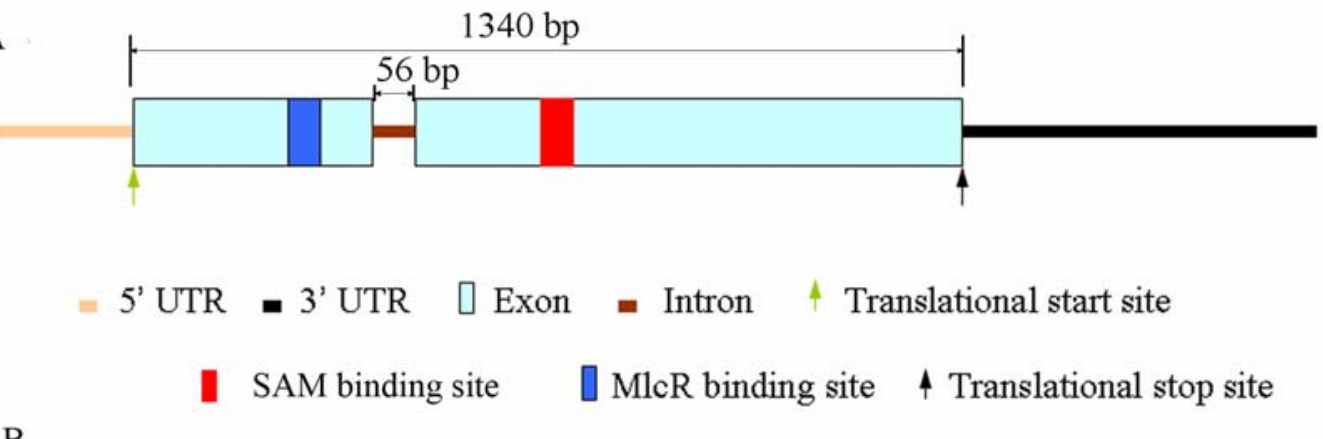

B

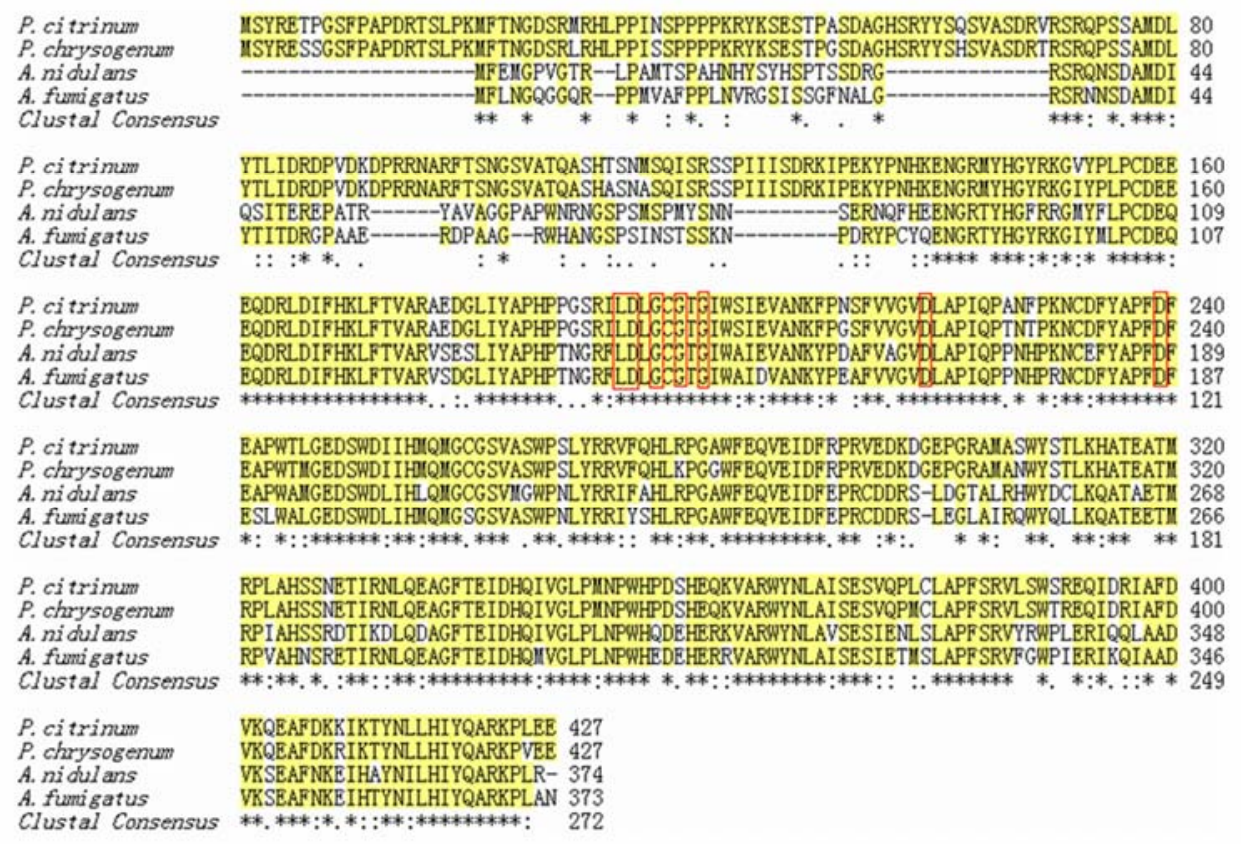

Figure 2 Overview of P. citrinum LaeA. (A) Schematic of laeA gene. The full-length Pci-laeA genomic DNA sequence is $1340 \mathrm{bp}$ in size, with a 56-bp intron spanning nucleotides 390-445 of Pci-laeA genomic DNA. (B) Amino acid comparison of P. citrinum, P. chrysogenum, A. nidulans and A. fumigatus LaeA proteins. GenBank Accession Numbers.: GU647101 for P. citrinum LaeA protein, CAP94071.1 for P. chrysogenum LaeA protein, AAQ95166.1 for A. nidulans LaeA protein, XP-752835.1 for A. fumigatus LaeA protein. The conserved SAM binding sites of protein methyltransferase are boxed and exist in all LaeA proteins. 